\title{
Lightning Monitoring Integrated System
}

\author{
Michell C. O. da Cruz ${ }^{1}$, Marcio N. G. Lopes ${ }^{1}$, Alen Vieira ${ }^{1}$, Flavio Santos ${ }^{1}$, Ricardo \\ A. Shinkai ${ }^{1}$, Daniele Nogueira ${ }^{1}$ Marissa B. Carvalho, Anderson F. de Nazaré \\ ${ }^{1}$ Centro Gestor e Operacional do Sistema de Proteção da Amazônia (CENSIPAM) - \\ Belém - PA - Brasil \\ \{michell.cruz, marcio.lopes, alen.vieira, flavio.santos, \\ ricardo.shinkai, daniele.nogueira, marissa.carvalho, \\ anderson.nazare\} @sipam.gov.br
}

\begin{abstract}
Lightning is a natural phenomenon and presents severe risks to people and animals, as well as affects several segments of the productive sector. A web-based lightning monitoring system has been developed to integrate different lightning detection systems, as well as to generate spatial and tabular data and products, capable of assisting specialists and decision makers. The system also allows combining lightning data with satellite images, increasing the capacity of analysis in near real time. This tool proved to be stable and efficient, with an intuitive interface that facilitates interaction with users.
\end{abstract}

\section{Introduction}

Lightning are natural and highly severe phenomena characterized by a high-tension current that can travel for a few kilometers [Uman, 1987]. Different sources of detection systems employ different technologies for lightning detection and location, which results in a diversity of accuracy and derived information. They can be complementary to each other, and that makes its joint analysis of extreme relevance for decision makers.

The lightning data from several sources have different formats with distinct temporal and spatial resolutions, as well as different measured parameters, which hinder a more comprehensive analysis by specialists and users in general. Besides that, few of these sources has a precise coverage on Amazon or provide free access to data [Marotti and Pinto, 2017].

In this sense, it is imperative to develop a platform that can integrate different sub-systems of lightning detection in a single interface to better qualify information and improve analytical ability, reflecting in the improvement of monitoring and analysis products, especially in conditions involving severe weather.

The lightning monitoring system was integrated as one of the operational modules of SipamHidro ${ }^{1}$ (Sistema Integrado de Monitoramento e Alerta Hidrometeorológico), which consists in a web platform equipped with tools for atmospheric and hydrological data analysis and monitoring and qualified information to support the decision making with focus in the Amazon. The lightning monitoring system aims to provide for free a near real time lightning monitoring that combines: supporting information as satellite; improved analysis by consulting and visualizing past data; and the generation of data and statistical reports.

1 Available in: http://siger.sipam.gov.br:8080/sipamhidro/publico/home.xhtml 


\section{Materials and Methods}

The monitoring system was developed as a client-server web service, wherein the client side is the Google Maps Application Programming Interface (API), which is responsible for creating and controlling the map, that displays all layer data, as well as JavaScript codes that communicate with the Java server to perform data requests. On the server side, it is operated as follows: the systems responsible for database requests; image repository; and layer generation for Google Maps through GeoServer, which constructs spatial data visualizations [Köbben, B. and Graham, M., 2009].

The database used is managed by PostgreSQL system with PostGIS spatial data extension, which supports the spatial data manipulation via SQL [Köbben, B. and Graham, M., 2009]. The use of PostgreSQL and PostGIS as well as others free software was to make the system less costly. To carry out the monitoring system development, initially it was only used lightning data from STARNET (Sferics Tracking and Ranging Network) network. In a next system version, it will be added data from GLM (Global Lightning Mapper) sensor of GOES-16 (Geostationary Operational Environmental Satellite) and the Lightning Detection Network of the Amazon Protection System (RDR-SIPAM).

STARNET operates on the Very Low Frequency (VLF) and consists of thirteen long-range terrestrial sensors, which are located in South America, the Caribbean, The United States and South Africa. The STARNET system detection is based on the Arrival Time Difference (ATD) of the sferics, which are noise in the VLF frequency produced by lightning [Rodriguez, C. et al. 2015]. It has a radius location error that can reach $12 \mathrm{~km}$ and its efficiency is not more than 50\%, capturing mostly cloud-ground lightning. However, it's sensors have a range that can pass of $5000 \mathrm{~km}$, allowing a good coverage of both the continent and the Brazilian coast. The information provided by STARNET are date/hour; geographic coordinates of the event; sensors participating in the solution; biggest solution error in distance (meters) and time (microseconds); polarity; number of sensors and ATD pairs used in the solution.

The database contains records of lightning since 2013 until nowadays, which are received at regular intervals of 5 minutes. The monitoring system access these data through requests to GeoServer (for the generation of map layers); and through an API for the generation of report files.

The platform also incorporated the synchronized availability of meteorological satellite GOES-16 images obtained through the GeonetCast Americas system (part of the global network of surface, atmospheric and metadata data dissemination system). The satellite data are received by a broadcast on a terrestrial station at intervals of 15 minutes in NetCDF (Network Common Data Form) files. These files are processed to generate PNG images through a Python script using the library GDAL (Geospatial Data Abstraction Library). Then, the images are made available for the monitoring system requests through API.

\section{Integrated System of Lightning Monitoring}

When the Lightning Module in SipamHidro is opened, the user has an interactive Google Maps API map, centered in the Legal Amazon with an expandable floating menu (Figure 1). This menu is subdivided between three main functionalities (monitoring, accumulated and report). In addition to these, there is an auxiliary functionality, which is responsible for inserting the satellite GOES-16 images on the 

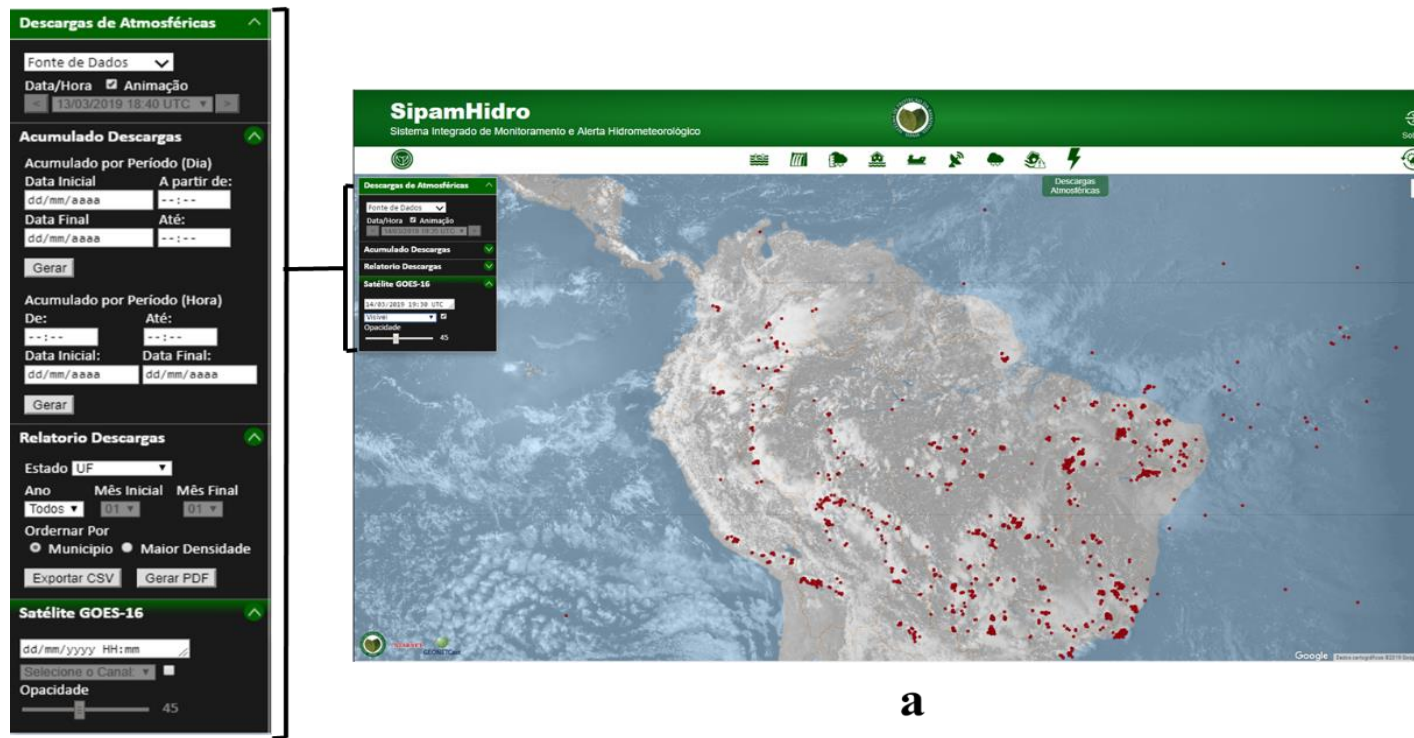

$\mathbf{a}$

b

Figure 1: Monitoring system main page with (b) the expansive float menu combining the lightning data from STARNET and the visible image of satellite GOES-16

map. Through parameters and filters controls, the user can interact with the features that configure the data visualization in the map.

Lightning occurrences are represented on the map by red spots at the incidence sites. Since many other data sources will be available, including different spatial coverage domains, the map allows the user to view the entire world map.

a) Lightning Monitoring: The monitoring function is responsible for displaying the lightning occurrences detected in the last hour in animated form and near real-time. Initially, the information is presented in the map in segmented layers and chronologically animated, which presents the accumulated lightning occurrences detected during the 5-minute interval, making it possible to infer the behavior pattern of electrical storms. These layers are updated every 5 minutes, thus keeping itself as updated as possible.

The lightning events visualizations can be synchronously combined with satellite imagery at different wavelengths (visible, infrared and water vapor), to increase the value to monitoring and expand analytical capabilities. Satellite images are received and updated at 15-minute intervals. When the animation is disabled, the user can select, move forward or rewind to any layers, which was previously animated. In addition, the

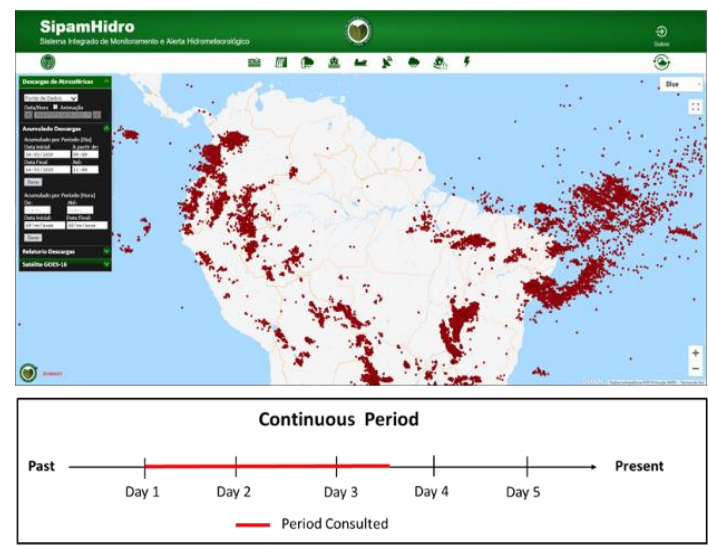

a

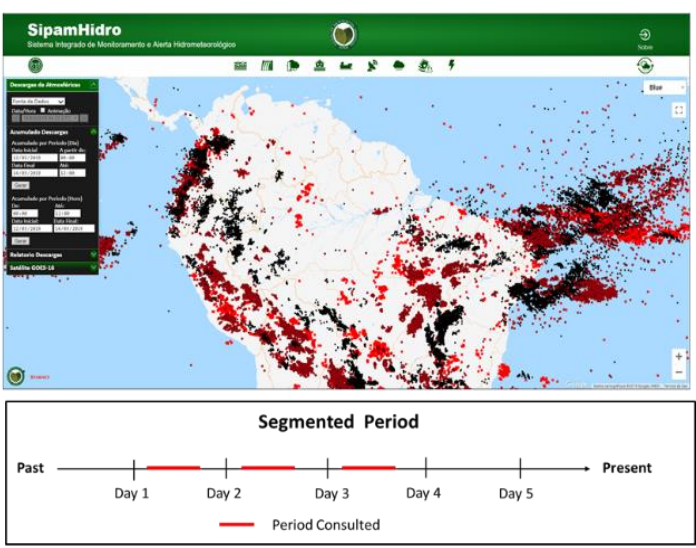

b

Figure 2: Types of accumulated lightning queries. (a) Continuous period; (b) Segmented period 
user can, at any time, select which sources will be exhibited on the map through an existing filter in the menu.

b) Accumulated Lightning: This functionality displays on the map all occurrences of lightning detected in a given time interval. In this function, the user can query a continuous (figure $2 a$ ) or segmented period (Figure $2 b$ ), considering a time interval of up to 3 consecutive days.

c) Lightning Data Reports: This function is intended to export the available lightning data in the system to standardized reports that allow a direct analysis or a tabular file format for manipulation by users in any spreadsheet calculation application for derived data generation, charts elaboration, among other possibilities. Thus, the system allows exporting data, both in PDF and CSV. For this, the user must set the year (from 2013 until present) and the desired state (belonging to the Legal Amazon). The current exported report model contains a monthly summary of lightning occurrence to all municipalities in the selected state and the annual sum of lightning density during the year surveyed. At first, data and products from other regions will be restricted for internal consultation. Future studies on accesses should mark the full availability of data.

\section{Conclusion}

The web system of lightning monitoring is an important tool and has proved to be very efficient in automating the process of integrating, requesting, making available and visualizing lightning data generated by different sources and hosted by Censipam (Centro Gestor e Operacional do Sistema de Proteção da Amazonia) at SipamHidro, as well as being able to filter such data according to selected parameters, in addition to generating the result in the format desired by the users in an intuitive way. The platform will facilitate Censipam's availability of past and near real time lightning data, allowing it to serve users from several productive sectors, such as energy, telecommunication, civil defense, among others. Another facility provided by the platform is the presentation of data combined with satellite images, improving the capacity for analysis and decision making about severe weather events.

\section{Acknowledgement}

This work was supported by the Centro Gestor e Operacional do Sistema de Proteção da Amazônia - Censipam and Conselho Nacional de Desenvolvimento Científico e Tecnológico - CNPq - Brazil. The authors would also like to thank the STORM-T Laboratory of the University of São Paulo-USP, the Instituto Nacional de Pesquisas Espaciais -INPE and the National Oceanic and Atmospheric Administration-NOAA for providing the data.

\section{References}

Marotti, A., and Pinto, O. (2017). Analysis of cloud-to-ground lightning density in Brazil. XIV International Symposium on Lightning Protection. IEEE.

Köbben, B. and Graham, M. (2009) Maps and mash-ups: the national atlas and Google Earth in a geodata infrastructure. 12th Agile international conference: Grid technologies for geospatial applications.

Rodriguez, C. et al. (2015) 9 years of lightning measurements in South America as detected by STARNET.XIII International Symposium on Lightning Protection. IEEE

Uman, M. A. (1987) The Lightning Discharge. San Diego: Academic Press, 377 p. 